\title{
Impact des déficits cognitifs dans les activités de la vie quotidienne des personnes souffrant de schizophrénie
}

\section{Impact of the cognitive deficits on the daily-life activities of people with schizophrenia}

\author{
M.-N. Levaux ${ }^{\mathrm{a}}$, J.-M. Danion ${ }^{\mathrm{b}}$ \\ ${ }^{a}$ Unité de Psychopathologie Cognitive de Liège, boulevard du Rectorat, B33, 4000 Liège, Belgique \\ ${ }^{\mathrm{b}}$ Unité Inserm 666 de Strasbourg, Pôle de Psychiatrie et de Santé Mentale, Service de Psychiatrie, Hôpitaux Universitaires de Strasbourg, \\ 67091 Strasbourg, France
}

\begin{abstract}
RÉSUMÉ
Il est actuellement bien établi que les dysfonctions cognitives contribuent de manière déterminante aux difficultés de la vie quotidienne, sociale et professionnelle des personnes présentant une schizophrénie. Les variables cognitives expliqueraient entre 16 et $30 \%$ de la variance enjeu dans le statut fonctionnel évalué de un à trois ans et demi plus tard. Cependant, la spécificité des relations complexes entre les domaines cognitifs et les variables fonctionnelles reste encore à identifier. À cette fin, il est nécessaire de conduire des études en respectant différents principes : choisir des tâches cognitives non multidéterminées ; explorer des tâches quotidiennes délimitées (e.g., faire ses courses, choisir un menu, préparer un repas), notamment par une observation en situation réelle afin d'identifier les composantes problématiques et cela, en établissant des hypothèses a priori quant à la nature cognitive des difficultés ; prendre en compte l'hétérogénéité des déficits cognitifs et des difficultés fonctionnelles des personnes souffrant de schizophrénie. Il reste également à investiguer la présence de variables médiatrices des relations, telles que le potentiel d'apprentissage, la cognition sociale, les processus métacognitifs et la motivation intrinsèque. Par ailleurs, d'autres facteurs personnels ou environnementaux peuvent aussi jouer un rôle dans le statut fonctionnel, comme par exemple les symptômes négatifs, la conscience qu'a la personne de son état et de ses conséquences ou l'attitude critique des proches envers la personne souffrant de schizophrénie.
\end{abstract}

Mots clés : Activités de la vie quotidienne ; Déficits cognitifs ; Schizophrénie

\begin{abstract}
It is now well-recognized that cognitive dysfonctions contribute to a decisive way to the difficulties of the everyday, social and professional life of the people with schizophrenia. The cognitive variables would explain between 16 and $30 \%$ of the variance in the functional status when assessed one to three and a half years later. However, the specificity of the complex relationships between the cognitive domains and the functional variables still remains to be identified. To this end, it is necessary to carry out studies by respecting various principles: selecting not multi-determined cognitive tasks; investigating specific daily-life tasks (e.g. shopping, choosing a menu, preparing a meal), in particular by an observation in real situation in order to identify the problematic components; this has to be made by determining a priori hypotheses regarding the cognitive nature of the difficulties; taking into account the heterogeneity of the cognitive deficits and the functional difficulties of people with schizophrenia. It also remains to investigate the presence of mediator variables of the relationships, such as the potential of learning, the social cognition, the metacognitive processes and the intrinsic motivation. Besides, other personal or environmental factors can also play a role in the functional status, as for example, the negative symptoms, the awareness that has the person of his(her) state and its consequences, or the critical attitude of relatives towards the person with schizophrenia.
\end{abstract}

Keywords: Cognitive deficits ; Daily-life activities ; Schizophrenia

L'exploration du fonctionnement cognitif semble fournir des informations essentielles concernant le devenir fonctionnel des personnes avec schizophrénie. En effet, il apparaît que ce devenir est davantage lié à la sévérité des déficits cognitifs qu'aux principaux symptômes cliniques de la schizophrénie. En tout état de cause, il est actuellement bien établi que les dysfonctions cognitives affectent le statut fonctionnel des personnes avec schizophrénie. Les premières revues de la littérature sur le sujet [8,9] ont examiné dans quelle mesure les troubles cognitifs pouvaient être reliés au statut fonctionnel. Green [8] a ainsi montré que la mémoire à long 
terme (épisodique) verbale était associée à trois domaines fonctionnels, à savoir l'acquisition d'habiletés sociales et instrumentales, la capacité de résolution de problèmes interpersonnels et le fonctionnement (social et professionnel) dans la communauté. La vigilance était corrélée à la résolution de problèmes et à l'acquisition d'habiletés sociales. Enfin, les fonctions exécutives étaient reliées à l'intégration dans la communauté. De plus, Green et al. [9] concluaient que ces mesures cognitives (y compris la mémoire immédiate verbale), dans leur ensemble, expliquaient 20 à $60 \%$ de la variance dans le statut fonctionnel. D'autres revues de questions [10,15] ont répertorié les études longitudinales portant sur les relations temporelles entre le fonctionnement cognitif et le statut fonctionnel (le statut professionnel, l'adaptation sociale et les activités de la vie quotidienne) chez les patients souffrant de schizophrénie. La performance cognitive initiale prédisait le statut fonctionnel subséquent (période pouvant s'étendre jusqu'à 12 années ; [10]) et des changements de performances cognitives étaient associés à un changement correspondant dans le statut fonctionnel des personnes sur le long terme (période pouvant s'étendre jusqu'à six années ; [15]).

Il apparaît cependant que le pouvoir prédicteur de la cognition sur le statut fonctionnel est relativement modéré et assez variable selon les études. Ainsi, dans un groupe de 40 personnes présentant une schizophrénie, Velligan et al. [25] ont mis en évidence que les variables cognitives évaluées (la mémoire verbale, les fonctions exécutives, la vigilance, la mémoire visuelle et les capacités d'organisation) expliquaient entre 16 et $30 \%$ de la variance enjeu dans les mesures du statut fonctionnel obtenues de un à trois ans et demi plus tard (selon les mêmes dimensions que celles définies par Green et al.). De plus, les auteurs n'ont relevé aucune spécificité dans les relations entre les domaines cognitifs évalués et les variables fonctionnelles prises en compte. Trois raisons peuvent être invoquées pour rendre compte de cette absence de spécificité. La première vient de la nature multidéterminée des tests classiquement utilisés pour évaluer le fonctionnement cognitif des personnes avec schizophrénie. De plus, ces tests cognitifs n'explorent pas certains processus spécifiquement en jeu dans les activités de la vie quotidienne, telle que la capacité de réaliser des tâches multiples (multitasking), du type « je dois me rendre au travail, organiser ma présentation pour cet après-midi et me souvenir d'aller chercher les enfants à l'école ». La réalisation de tâches multiples dépend de façon cruciale de la capacité de mémoire prospective, de la capacité à désengager et engager l'attention « au bon moment », ainsi que de la capacité à allouer de façon flexible les ressources attentionnelles vers les stimuli internes (les plans de réalisation de la tâche) et les stimuli externes [4].

Une autre raison pouvant expliquer le manque de spécificité observé dans les relations entre le fonctionnement cognitif et les variables fonctionnelles tient à la présence de variables médiatrices et/ou modératrices de cette relation. Green et al. [9] ont suggéré que le «potentiel d'apprentissage » (l'estimation dynamique de ce que la personne est capable d'apprendre et qu'il faut distinguer de l'évaluation statique de ce que la personne connaît actuellement) pourrait jouer un rôle intermédiaire entre la cognition et l'acquisition d'habiletés fonctionnelles. La contribution de certains processus de « cognition sociale » comme facteur limitant du changement fonctionnel dans la communauté (professionnel, social et de vie indépendante) a également été soulignée par Brekke et al. [3]. Koren et al. [13] mettent l'accent, quant à eux, sur l'importance des " processus métacognitifs », d'autorégulation et d'autocontrôle, qu'ils considèrent comme des déterminants fondamentaux du fonctionnement dans les activités de la vie quotidienne. La motivation intrinsèque (le désir de s'engager dans une activité d'apprentissage parce qu'elle est, de manière inhérente, intéressante et engageante) représente un autre mécanisme essentiel pouvant intervenir entre le statut cognitif et le fonctionnement psychosocial [19].

Enfin, une troisième raison permettant de rendre compte du manque de spécificité dans les relations entre statut fonctionnel et cognition tient au fait que le statut fonctionnel est essentiellement mesuré à l'aide de questionnaires globaux incluant un grand nombre de variables, telles que les relations familiales, la vie indépendante et le statut professionnel. Ces mesures globales ne permettent pas de distinguer les différents domaines fonctionnels (les activités de la vie quotidienne, le statut professionnel, les rôles sociaux), lesquels font sans aucun doute, pour une part au moins, appel à des compétences cognitives spécifiques.

Les limites du pouvoir prédicteur de l'évaluation cognitive sur l'évolution fonctionnelle des personnes présentant une schizophrénie ont conduit à l'élaboration d'outils visant à évaluer de façon plus directe le comportement des personnes dans des activités de la vie quotidienne. Une des mesures les plus utilisées actuellement, l'University of California, San Diego Performance-Based Skills Assessment (UPSA [21]), évalue la capacité des personnes présentant une schizophrénie à réaliser des tâches de la vie quotidienne dans cinq domaines, comme par exemple la gestion des finances (la personne doit remplir un chèque pour payer une facture), ou la planification d'une activité récréative (la personne doit s'imaginer qu'elle va visiter un zoo et lire un texte qui décrit les heures d'ouverture du zoo, les attractions animalières possibles et le temps qu'il fera ; elle doit ensuite répondre à quelques questions sur le texte et identifier cinq objets à emporter dans le but de passer la journée au zoo). Ces outils se sont avérés être des mesures plus directes et valides du fonctionnement quotidien par rapport aux mesures plus distales représentées par les tests cognitifs [12,21]. Cependant, différentes critiques ont été adressées à ces outils standardisés d'évaluation dans des situations proches (des " simulations ») de la vie 
quotidienne [16,18]. En particulier, il apparaît que la validité prédictive de ces mesures concernant le fonctionnement dans le monde réel n'a pas encore été solidement démontrée. En fait, les exigences de l'évaluation dans ces situations de «simulations » d'activités de la vie quotidienne diffèrent sur de nombreux points de celles rencontrées dans les activités du monde réel [17]. La validité prédictive des évaluations en situation de « simulation » peut être affectée par les comportements sélectionnés pour l'évaluation (par exemple, dans l'UPSA, le domaine des transports ne sera reflété que par la capacité à comprendre une carte reprenant les différents trajets des bus), le détail des consignes explicites fournies (qui ne sont généralement pas présentes dans le monde réel), l'environnement artificiel du testing et la capacité de la personne à jouer un rôle dans des mises en situations réelles (par exemple, lors de l'évaluation des capacités sociales à l'aide de l'outil SSPA, la personne doit jouer le rôle d'une personne qui rencontre un voisin nouvellement installé dans l'immeuble). De plus, ces outils proposent des situations qui ne représentent pas vraiment la complexité (le caractère multitasking) de nombreuses activités de la vie quotidienne. Par exemple, dans l'UPSA, le domaine des « tâches ménagères » est représenté par une seule tâche simple de lecture d'une recette et d'élaboration d'une liste de courses.

Une autre manière d'évaluer le fonctionnement dans le monde réel réside dans l'observation directe de l'exécution d'une tâche quotidienne. Cette méthode d'évaluation (avec grille de cotation du comportement) permet d'examiner en détail un domaine fonctionnel altéré et d'identifier directement les composantes problématiques. Ainsi, quelques études se sont penchées sur l'évaluation des performances de personnes avec un diagnostic de schizophrénie dans des activités spécifiques de la vie quotidienne, telles que faire ses courses, choisir un menu ou préparer un repas $[1,7,22,23]$. Ces études ont permis de mettre en évidence des corrélations significatives entre des difficultés spécifiques à accomplir ces tâches de la vie quotidienne (des omissions, des commissions, des répétitions ou des erreurs de séquences dans les actions) et des déficits cognitifs particuliers (exécutifs, mnésiques, attentionnels).

Par exemple, Semkovska et al. [23] ont exploré chez 27 personnes souffrant de schizophrénie, comparées à 27 personnes de contrôle, les relations entre la réalisation directe de trois activités de la vie quotidienne et des mesures cognitives (évaluant la mémoire épisodique verbale et visuelle, l'attention et les fonctions executives). Les activités de la vie quotidienne consistaient à : sélectionner un menu constitué d'une entrée, d'un plat et d'un dessert (sur la base d'ingrédients placés sur une table et de recettes qui différaient selon la disponibilité des ingrédients, le prix des ingrédients manquants et le temps de préparation); acheter des ingrédients pour le menu sur base d'une liste et en dépensant le moins d'argent possible ; préparer un repas avec entrée, plat principal et dessert en une heure (les trois composants du menu devant être prêts au même moment). Les analyses de la performance étaient effectuées par deux juges indépendants sur base d'enregistrements vidéo en se fondant sur une séquence optimale préétablie. Les résultats montrent que les fonctions exécutives, particulièrement la flexibilité (ou capacité à désengager son attention durant la réalisation d'une activité), étaient le facteur le plus associé (après contrôle de la vitesse de traitement) à la réalisation des tâches, le lien le plus important concernant la situation la plus complexe (de multitasking), à savoir la préparation du repas. Les symptômes négatifs étaient également corrélés aux erreurs commises durant la réalisation des tâches (omissions durant le choix du menu ; répétitions et erreurs de planification durant la préparation d'un repas). De manière générale, les limites de ces études sont d'avoir utilisé des tests cognitifs multidéterminés et d'avoir été menées sans hypothèse a priori sur la nature des mécanismes cognitifs censés être impliqués dans la réalisation des différentes tâches de la vie quotidienne. De plus, ces études ne prennent pas en compte l'hétérogénéité des déficits cognitifs et des difficultés fonctionnelles des personnes souffrant de schizophrénie [5,20,24].

En résumé, afin de mieux comprendre la nature des relations complexes entre les domaines cognitifs et les variables fonctionnelles chez les personnes souffrant de schizophrénie, il est nécessaire de conduire des études en respectant différents principes :

- choisir des tâches cognitives non multidéterminées et plus pertinentes sur le plan théorique ;

- explorer des tâches quotidiennes délimitées (e.g., faire ses courses, choisir un menu, préparer un repas), notamment par une observation en situation réelle afin d'identifier les composantes problématiques, cela en établissant des hypothèses a priori quant à la nature cognitive des difficultés ;

- prendre en compte l'hétérogénéité des déficits cognitifs et des difficultés fonctionnelles des personnes souffrant de schizophrénie.

Dans une perspective de remédiation cognitive, ces différents principes seront extrêmement utiles à prendre en compte pour l'élaboration d'un programme thérapeutique basé sur les préoccupations quotidiennes de la personne concernée et sur des hypothèses quant à la nature des mécanismes qui soustendent les difficultés rencontrées par celle-ci dans sa vie quotidienne. Il reste également à investiguer la présence de variables médiatrices des relations, telles que le potentiel d'apprentissage, la cognition sociale, les processus métacognitifs et la motivation intrinsèque. Par ailleurs, d'autres facteurs personnels ou environnementaux peuvent aussi jouer un rôle dans le 
statut fonctionnel, comme par exemple les symptômes négatifs [2,11 ], la conscience qu'a la personne de son état et de ses conséquences [14] ou l'attitude critique des proches envers la personne avec schizophrénie [6[.

\section{Conflit d'intérêt}

Aucun des auteurs n'a de conflit d'intérêts.

\section{Références}

[1] Aubin G, Stip E, Gélinas I, Rainville C, Chapparo C. Daily activities, cognition and community functioning in persons with schizophrenia. Schizophr Res 2009;107:313-8.

[2] Bowie CR, Reichenberg A, Patterson TL, Heaton RK, Harvey PD. Determinants of real-world functional performance in schizophrenia subjects: correlations with cognition, functional capacity, and symptoms. Am J Psychiatry 2006;163:418-25.

[3] Brekke JS, Hoe M, Long J, Green MF. How neurocognition and social cognition influence functional change during community-based psychosocial rehabilitation for individuals with schizophrenia. Schizophr Bull 2007;33: 1247-56.

[4] Burgess PW, Simons JS, Dumontheil I, Gilbert SJ. The gateway hypothesis of rostral PFC function. In: Duncan J, Phillips L, McLeod P, editors. Measuring the mind: speed control and age. Oxford University Press; 2005. p. 215-46.

[5] Chan RCK, Chen EYH, Cheung E, Chen R, Cheung H. The components of executive functioning in a cohort of patients with chronic schizophrenia: a multiple single-case study design. Schizophr Res 2006;81:173-89.

[6] Girón M, Gómez-Beneyto M. Relationship between family attitudes and social functioning in schizophrenia: a nine-month follow-up prospective study in Spain. J Nerv Ment Dis 2004;192:414-20.

[7] Godbout L, Limoges F, Allard I, Braun CM, Stip E. Neuropsychological and activity of daily living script performance in patients with positive or negative schizophrenia. Compr Psychiatry 2007;48:293-302.

[8] Green MF. What are the functional consequences of neurocognitive deficits in schizophrenia? Am J Psychiatry 1996;153:321-30.

[9] Green MF, Kern RS, Braff DL, Mintz J. Neurocognitive deficits and functional outcome in schizophrenia: are we measuring the 'right stuff? Schizophr Bull 2000;26:119-36.

[10] Green MF, Kern RS, Heaton RK. Longitudinal studies of cognition and functional outcome in schizophrenia: implications for MATRICS. Schizophr Res 2004;72:41-51.

[11] Greenwood KE, Landau S, Wykes T. Negative symptoms and specific cognitive impairments as combined targets for improved functional outcome within cognitive remediation therapy. Schizophr Bull 2005;31:910-21.

[12] Harvey KE, Velligan DI, Bellack AS. Performance-based measures of functional skills: usefulness in clinical treatment studies. Schizophr Bull 2007;33:1138-48.

[13] Koren D, Seidman L, Goldsmith M, Harvey PD. Real-world cognitive-and metacognitive-dysfunction in schizophrenia: a new approach for measuring (and remediating) more 'right stuff. Schizophr Bull 2006;32:310-26.

[14] Lysaker PH, Bryson GJ, Bell MD. Insight and work performance in schizophrenia. J Nerv Ment Dis 2002;190:142-6.

[15] Matza LS, Buchanan R, Purdon S, Brewster-Jordan J, Zhao Y, Revicki DA. Measuring changes in functional status among patients with schizophrenia: the link with cognitive impairment. Schizophr Bull 2006;32:666-78.

[16] Mausbach BT, Moore R, Bowie CR, Cardenas V, Patterson TL. A review of instruments for measuring functional recovery in those diagnosed with psychosis. Schizophr Bull 2009;35:307-18.

[17] McKibbin CL, Brekke JS, Sires D, Jeste DV, Patterson TL. Direct assessment of functional abilities: relevance to persons with schizophrenia. Schizophr Res 2004;72:53-67.

[18] Moore DJ, Palmer BW, Patterson TL, Jeste DV. A review of performance-based measures of functional living skills. J Psychiatr Res 2007;41:97-118.

[19] Nakagami E, Xie B, Hoe M, Brekke JS. Intrinsic motivation, neurocognition, and psychosocial functioning in schizophrenia: testing mediator and moderator effects. Schizophr Res 2008;105:95-104.

[20] Palmer BW, Heaton RK, Gladsjo JA, Evans J, Patterson TL, Golshan S, et al. Heterogeneity in functional status among older outpatients with schizophrenia: employment history, living situation, and driving. Schizophr Res 2002;55:205-15.

[21] Patterson TL, Goldman S, McKibbin CL, Hughs T, Jeste DV. UCSD Performance-Based Skills Assessment: development of a new measure of everyday functioning for severely mentally ill adults. Schizophr Bull 2001;27:235-45.

[22] Rempfer MV, Hamera EK, Brown CE, Cromwell RL. The relations between cognition and the independent living skill of shopping in people with schizophrenia. Psychiatry Res 2003;117:103-12.

[23] Semkovska M, Bédart M, Godbout L, Limoge F, Stip E. Assessment of executive dysfunction during activities of daily living in schizophrenia. Schizophr Res 2004;69:289-300.

[24] Shallice T, Burgess PW, Frith CD. Can the neuropsychological case-study approach be applied to schizophrenia? Psychol Med 1991;21:661-73.

[25] Velligan DI, Bow-Thomas CC, Mahurin RK, Miller AL, Halgunseth LC. Do specific neurocognitive deficits predict specific domains of community function in schizophrenia? J Nerv Ment Dis 2000;190:142-6. 


\section{Discussion}

Dr Charbit - Les difficultés cognitives dans la schizophrénie sont-elles comparables à celles observées dans l'autisme?

Réponse du rapporteur - Nous pouvons retrouver des domaines cognitifs communément déficitaires dans ces deux populations, tels que, par exemple, les fonctions exécutives (e.g., initiation de la réponse, flexibilité, inhibition, planification), la vitesse de traitement de l'information ou les processus touchant la cognition sociale (e.g., reconnaissance des émotions, capacité à prendre en compte le point de vue de l'autre et ses intentions, résolution de problèmes sociaux, etc.). Nous pouvons également identifier un autre point commun concernant le profil cognitif de personnes souffrant de schizophrénie et celui des personnes souffrant d'autisme, celui de l'hétérogénéité cognitive inter- et intra-individuelle. En effet, le profil des fonctions cognitives trouvées déficitaires et préservées diffère d'une personne à l'autre, dans la schizophrénie comme dans l'autisme. Ainsi, une étude récente réalisée par Togwood et collaborateurs en 2009 a mis en évidence une variabilité intra-individuelle au sein de profils de personnes présentant un autisme, avec des performances cognitives trouvées sous- et supranormales selon les domaines cognitifs évalués.

Dr Van Amerongen - Dans toutes ces études, il faut veiller à ne pas mélanger les patients schizophrènes, schizoaffectifs, voire les psychotiques. Il faut savoir à qui l'on s'adresse.

Au plan méthodologique, il faut dans ces études préciser la situation, le statut social « hospitalisé ou pas ».

Réponse du rapporteur - Il est effectivement important dans les études empiriques de constituer des groupes de personnes présentant des caractéristiques communes dont on fait l'hypothèse qu'elles peuvent exercer d'une manière ou d'une autre une influence sur les résultats. En corollaire, il faut préciser les critères de l'échantillon que l'on étudie afin de faciliter la généralisation des résultats aux personnes présentant ces mêmes caractéristiques. Cela peut concerner, selon l'objet d'intérêt, le diagnostic, la situation ou le statut de vie indépendante, ainsi que beaucoup d'autres caractéristiques pouvant influencer les résultats. En effet, la schizophrénie est un état psychopathologique associé à des dysfonctionnements psychologiques complexes qui impliquent de nombreux processus en interaction (endogènes et environnementaux) et qui peuvent ainsi s'exprimer de façon très variée et individualisée. Aussi, considérant cette grande hétérogénéité cognitive et fonctionnelle qui caractérise la schizophrénie, il pourrait être fructueux de mener des études selon une méthodologie d'analyse en cas multiples qui permettrait d'investiguer la complexité des profils rencontrés.

Dr Ropert - Merci à $\mathrm{M}^{\text {me }}$ Levaux pour la clarté et la « lisibilité » de sa communication. Il me semble important qu'elle ait insisté sur la notion de «flexibilité »: celle-ci constitue en effet un indice révélateur très sensible, d'ailleurs non spécifique de la schizophrénie, ni même du groupe des psychoses dans leur ensemble. À l'occasion d'une longue pratique des « visites à domicile », effectuées en compagnie d'un assistant social chez des sujets souffrant d'un début de déficit cognitif (surtout mémoriel) - dans le cadre de l'application de la loi sur les « incapables majeurs » -nous avions remarqué que le désarroi de ces sujets portait non seulement sur les «oublis» quotidiens (dont témoignaient d'évidence les multiples « aide-mémoires » affichés dans leur logement) mais bien davantage, et de façon très sensible, par leurs réactions de véritable désarroi quand un élément imprévu était venu interférer et modifier les plans qu'ils avaient établis pour régler leurs activités quotidiennes (organisation d'un «plan de repas », par exemple). Il faudrait sans doute étendre davantage cette étude de la notion de " flexibilité », dans l'appréciation des troubles des activités révélateurs, dans toute une série de manifestations psychopathologiques étudiées au long cours.

Réponse du rapporteur - Effectivement, dans l'étude de Semkovska et al. (2004) qui a exploré chez des personnes souffrant de schizophrénie les relations entre la réalisation directe de trois activités de la vie quotidienne (sélectionner un menu, faire les courses et préparer un repas) et des mesures cognitives, la capacité à désengager son attention d'une tâche à l'autre était le facteur le plus associé à la réalisation de l'activité de cuisine. Les processus cognitifs tout particulièrement impliqués dans les situations de la vie quotidienne, comme la capacité de s'adapter à des situations de type "multitâches » ou la capacité d'allouer de façon flexible son attention entre différentes tâches ou entre les informations internes (plans d'actions, pensées, émotions) et les informations externes, doivent faire l'objet d'études plus approfondies au sein de différents états psychopathologiques. Cette perspective s'inscrit dans une approche transdiagnostique qui suscite de plus en plus d'intérêt dans la littérature scientifique et qui met l'accent sur l'étude de processus psychologiques (dont les fonctions cognitives) à travers différents diagnostics. 